\title{
Bioproduction of fumaric acid: an insight into microbial strain improvement strategies
}

\author{
Joseph Sebastian ${ }^{\mathrm{a}}$, Krishnamoorthy Hegde ${ }^{\mathrm{a}}$, Pratik Kumar ${ }^{\mathrm{a}}$, Tarek Rouissi ${ }^{\mathrm{a}}$ and Satinder Kaur Brar ${ }^{\mathrm{a}, \mathrm{b}}$ \\ ${ }^{a}$ INRS-ETE, Université du Québec, Québec, Canada; ${ }^{b}$ Department of Civil Engineering, Lassonde School of Engineering, York University, \\ Toronto, Ontario, Canada
}

\begin{abstract}
Fumaric acid (FA), a metabolic intermediate, has been identified as an important carbohydrate derived platform chemical. Currently, it is commercially sourced from petrochemicals by chemical conversion. The shift to biochemical synthesis has become essential for sustainable development and for the transition to a biobased economy from a petroleum-based economy. The main limitation is that the concentrations of FA achieved during bioproduction are lower than that from a chemical process. Moreover, the high cost associated with bioproduction necessitates a higher yield to improve the feasibility of the process. To this effect, genetic modification of microorganism can be considered as an important tool to improve FA yield. This review discusses various genetic modifications strategies that have been studied in order to improve FA production. These strategies include the development of recombinant strains of Rhizopus oryzae, Escherichia coli, Saccharomyces cerevisiae, and Torulopsis glabrata as well as their mutants. The transformed strains were able to accumulate fumaric acid at a higher concentration than the corresponding wild strains but the fumaric acid titers obtained were lower than that reported with native fumaric acid producing $R$. oryzae strains. Moreover, one plausible adoption of gene editing tools, such as Agrobacterium-mediated transformation (AMT), CRISPR CAS-9 and RNA interference (RNAi) mediated knockout and silencing, have been proposed in order to improve fumaric acid yield. Additionally, the introduction of the glyoxylate pathway in $R$. oryzae to improve fumaric acid yield as well as the biosynthesis of fumarate esters have been proposed to improve the economic feasibility of the bioprocess. The adoption of some of these genetic engineering strategies may be essential to enable the development of a feasible bioproduction process.
\end{abstract}

\section{ARTICLE HISTORY}

Received 23 July 2018

Revised 18 April 2019

Accepted 30 April 2019

\section{KEYWORDS}

Fumaric acid;

bioproduction; genetic modification; metabolic engineering; transformation; $R$. oryzae; fumaric acid ester

\section{Introduction}

Microorganisms can produce a diverse range of chemicals and materials. These products of microbial metabolism, such as organic acids, have a wide range of applications and can be used in our daily life. Humans have a long history of reliance on microorganisms to produce diverse products ranging from chemicals, such as ethanol and organic acids, to consumables, such as bread, yogurt, and cheese. Fumaric acid (FA), also referred to as (E)-2-butenedioic acid or trans-1,2ethylene dicarboxylic acid, is one such microbial metabolic products that have been identified as a platform chemical by the US Department of Energy (DOE) in $2004[1,2]$. It is as an important carbohydrate derived platform chemical, a key intermediate of microbial metabolism, with several applications in the synthesis of bio-based chemicals and polymer production. The diverse applications are rendered due to the presence of the dicarboxylic groups. This makes FA amenable to chemical modifications and being suitable for use as a monomer for the synthesis of biodegradable polymers [3].

FA is mainly used as a food additive and food acidulant and in the production of paper resins, unsaturated polyester resins, and plasticizers [4-6]. The application of fumaric acid, in general, has been reviewed extensively by Das et al. $[7,8]$ and the diverse industrial applications of this acid as a resin, plasticizer, and component for polymer synthesis has been reviewed by Doscher et al. [9]. More recently, derivatives of FA, especially fumaric acid esters (FAEs) have found its application as an important chemical with a wide array of biomedical applications, such as psoriasis and sclerosis treatment and a support material for tissue engineering. Clinical level studies on the pharmacological effects of FA and its ester derivatives (FAEs)

CONTACT Satinder Kaur Brar $\otimes$ satinder.brar@ete.inrs.ca, Satinder.Brar@lassonde.yorku.ca $\mathbf{\theta}$ Institut national de la recherche scientifique, Centre Eau, Terre \& Environnement, 490 de la Couronne, Québec (Qc) G1K 9A9, Canada 
have confirmed their efficacy. Methyl esters of fumaric acid, mono-methyl fumarate (MMF) and dimethyl fumarate (DMF) esters, has been even licensed for use in Europe for the treatment of severe psoriasis vulgaris $[10,11]$. The recent approval of FDA of DMF (Tecfidera) for use in the treatment of relapsing forms of multiple sclerosis, proves the importance of FA and FAEs. In addition, FAEs, specifically their ethyl esters, has found its application in tissue engineering as a scaffold as well as for drug delivery $[12,13]$. The review by Das et al. [8] provides comprehensive information about the diverse medical applications of fumaric acid and its derivatives.

The diverse applications of FA have fueled the demand for dicarboxylic acid, which is fulfilled by the means of chemical synthesis from petroleum products. Emphasis on the transition towards the biobased economy has led to the recognition of interest in the development of bioproduction strategies for FA production. FA is a key intermediate of the metabolic pathway of a diverse group of microorganisms and has led to the investigation of these microorganisms as a potential means of FA production. Fungal strains such as Rhizopus oryzae, has been identified as a FA overproducer and a potential source for FA. This review discusses various strain improvement strategies to improve $R$. oryzae mediated FA production as well as metabolic and genetic engineering strategies investigated to achieve microbial production using non-FA producers. These investigations have not led to commercial bioproduction of FA and the plausible reasons for this have been provided. Moreover, the advancement in metabolic engineering techniques have provided us with novel tools and possible avenues for the use of these strategies for the bioproduction of FA and its derivatives. Previous reviews have emphasized primarily on the control of process parameters in order to improve and control FA production. These reviews have provided little discussion on genetic engineering strategies that can be adopted to improve FA production. This lack of compiled information on strain improvement strategies, especially genetic engineering, is attempted to be considered in this review.

\section{Current status of the commercial synthesis of fumaric acid}

At present, the global demand for FA is solely fulfilled by petrochemical methods of synthesis as represented in Figure 1. It is derived by the isomerization of maleic acid obtained from hydrolysis of maleic anhydride.
The maleic anhydride is obtained by the oxidation of butane or benzene in the presence of the catalyst, vanadyl pyrophosphate $[3,4]$. The catalyst mediated conversion is the only feasible technique for the selective conversion of butane into maleic acid [14]. This chemical catalyst mediated process provides a high process yield of FA $(112 \% \mathrm{w} / \mathrm{w})$ from maleic anhydride $[3,14]$. However, this process also leads to the formation of the toxic gas, carbon monoxide, and greenhouse gas, carbon dioxide, as byproducts, which in turn leads to environmental pollution and contributes to global warming [7]. The global demand for FA is expected to expand to over 300 Kilotons by the year 2020 from 225.2 Kilotons (2012). This increase in demand is mainly fueled by a rise in the demand for the acid used in the food and beverage industry, which accounts for over $35 \%$ of global consumption [15-17].

Moreover, FA is an important intermediate in the synthesis of edible products, such as L-malic acid and $L$-aspartic acid. The increase in demand for artificial sweeteners, such as aspartame, in beverages and foods, will further increase the worldwide demand for FA and its derivatives [18]. Furthermore, the recent identification of its pharmaceutical properties can lead to increased usage of FA and its derivatives in the pharmaceutical industry, as discussed earlier. This will further enhance the global demand for this acid. Additionally, the high price of crude oil, its depletion and the recent emphasis on green chemistry for sustainable development, has necessitated the adoption of alternate means for increased FA production $[15,19]$.

\section{Bioproduction of fumaric acid}

Microbial bioproduction of this carbohydrate derived biochemical provides an attractive alternative which ensures sustainable development by reducing the dependence on fossil fuels. Moreover, the use of renewable feedstocks helps the recovery of valuable carbon. FA is one such compound synthesized by many microorganisms, especially aerobic microbes, as an intermediate compound during the TCA cycle $[7,20]$. As early as 1911 the fungal strain, Rhizopus nigricans, was identified for its ability to synthesize FA as early as 1911. Later studies, involving multiple fungal species, concluded that Rhizopus species was the best producer of fumaric acid. Xu et al. [5] provided a brief summary of the history of the bioproduction of FA. Additionally, the adoption of a bioproduction strategy 

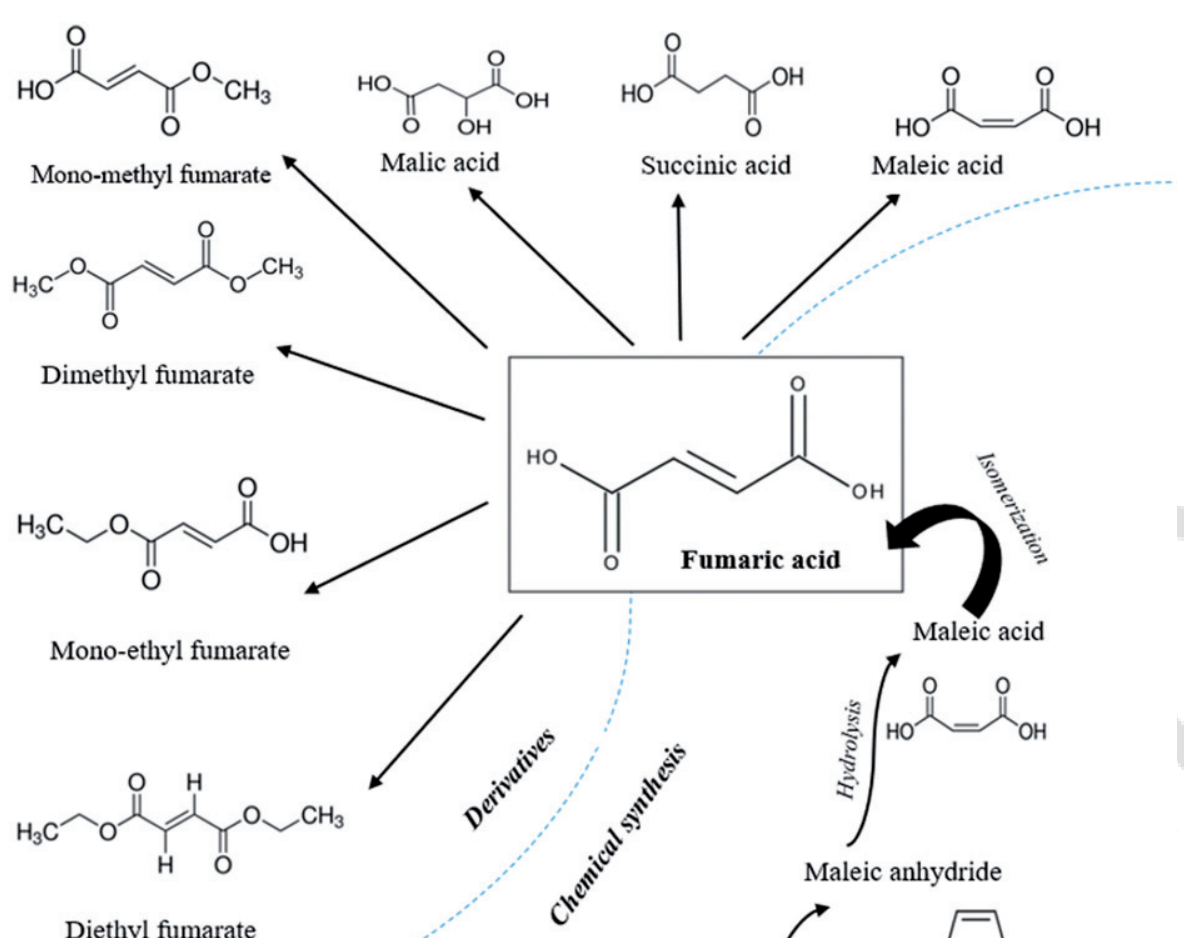

Diethyl fumarate

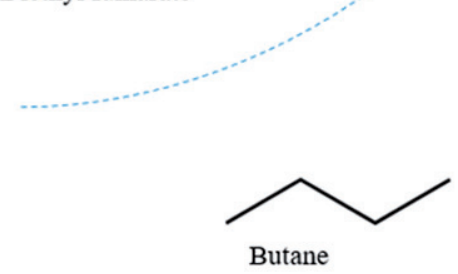

OR

Figure 1. Chemical fumaric acid synthesis and its derivatives.
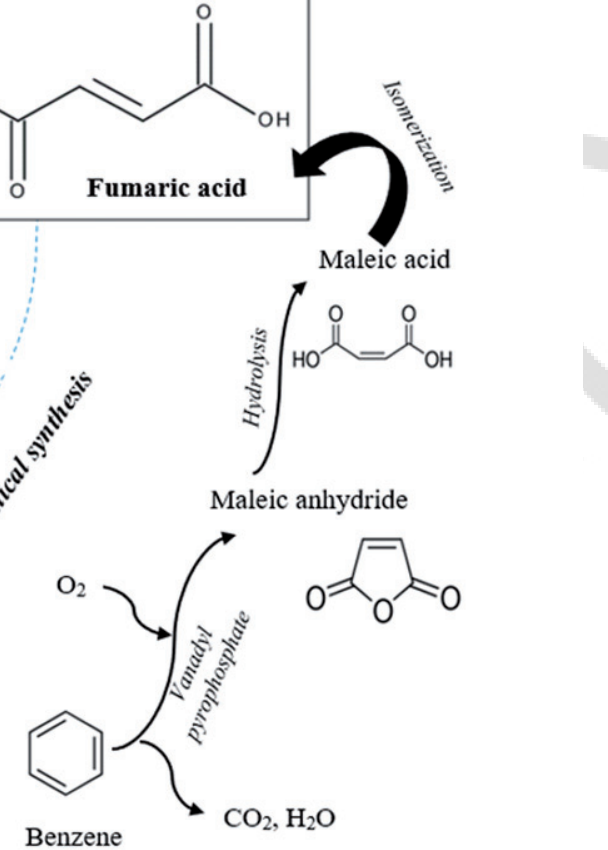

allows for carbon dioxide fixation and recovery, especially when organic industrial residues are employed.

However, the advent of less expensive petrochemical means of fumaric acid synthesis led to the discontinuation of the bioproduction of FA. Higher yields of FA are obtained during the chemical process $(112 \%$ $\mathrm{w} / \mathrm{w}$ against $85 \% \mathrm{w} / \mathrm{w}$ via fermentation using glucose as carbon source). Also, the lower production cost and better control over production conditions led to the widespread use of the chemical process for FA production [3]. The recent increase in the price of petroleum products, increasing the emphasis on low carbon footprint production strategies and green chemistry approaches for sustainable development. This has resulted in the resurgence of interest in fumaric acid production by fermentation $[5,15]$. Moreover, the adoption of bioproduction strategy allows for the utilization of renewable biomass as well as industrial residues for FA production. This in turn significantly reduces the production costs thereby improving its feasibility. Additionally, the low solubility of FA, 5-7 g/L (at room temperature), ensures its easy recovery by simple precipitation following acidification. Hence, no specialized strategies are required for the recovery of FA $[21,22]$. The FA crystals so recovered have been observed to be of high purity, with inorganic components in the fermentation medium being the primary contaminant, and can be used thereafter for low purity applications such as the manufacture of resins or polymers as well as cattle feed supplements [3]. To further improve FA purity, the use of activated carbon has been investigated and found to be effective $[23,24]$. The recovered FA (of purity $>99 \%$ ) can be used for biomedical applications such as psoriasis and multiple sclerosis treatment.

\section{Metabolic pathway of fumaric acid synthesis}

Fumaric acid is an important intermediate of the tricarboxylic acid (TCA) cycle (Krebs cycle). Two different metabolic pathways are mainly involved in the synthesis of FA viz. oxidative TCA cycle and reductive TCA pathway. Fixation of $\mathrm{CO}_{2}$ and conversion of pyruvate to oxaloacetate occurs during the TCA cycle. Reductive
296

297

298

299

300

301

302

303

304

305

306

307

308

309

310

311

312

313

314

315

316

317

318 


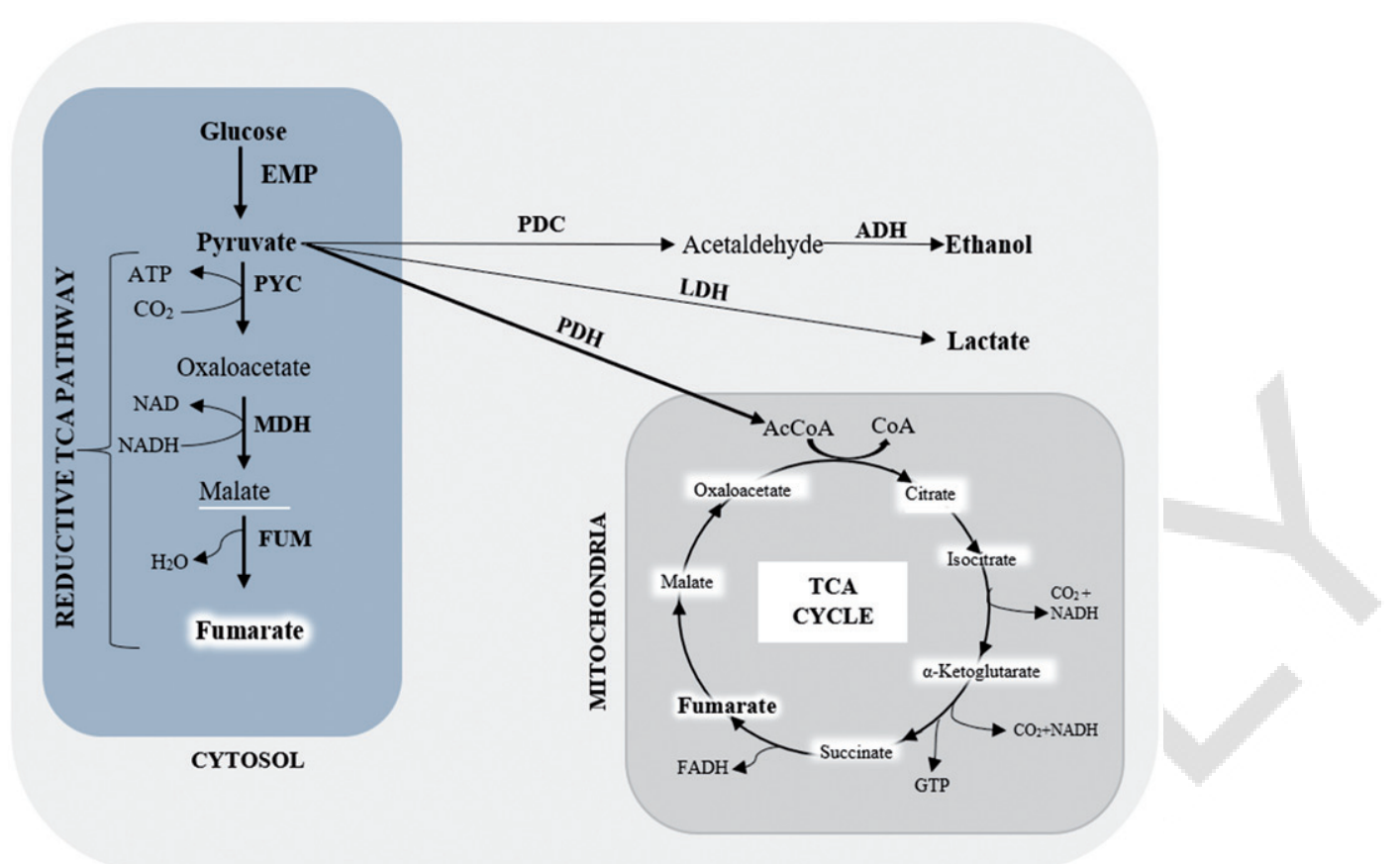

Figure 2. Overview of metabolic pathway and enzymes involved in the fumaric acid synthesis in $R$. oryzae. EMP stands for Embden-Meyerhof-Parnas pathway or glycolysis. The enzymes involved in the various steps of the pathway are PDC-pyruvate decarboxylase (EC 4.1.1.1); $\mathrm{ADH}$-alcohol dehydrogenase (EC 1.1.1.1); LDH—lactate dehydrogenase (EC 1.1.1.27); PDH—PYRUVATE dehydrogenase (EC 1.2.4.1); PYC - pyruvate carboxylase (EC 6.4.1.1); $\mathrm{MDH}$-malate dehydrogenase (EC 1.1.1.37) and FUMfumarase (EC 4.2.1.2). For color version of the figure refer e-print.

$\mathrm{CO}_{2}$ fixation catalyzed by the pyruvate carboxylase enzyme is the main reason for the accumulation of fumaric acid during fermentation as it was observed in the case of the Rhizopus species [5]. The reductive cycle has a maximal theoretical yield of 2 moles of FA per mole of glucose consumed and 2 moles of $\mathrm{CO}_{2}$ fixed. Reliance on reductive pyruvate carboxylation, as the sole pathway, will lead to no ATP formation. This ATP is required for cell maintenance and will affect overall cell growth. Hence, the TCA cycle is active during FA production and complete theoretical fumaric yield will not be achieved $[3,7]$. Adoption of strategies to limit fungal growth has the potential to ensure optimum fumaric acid production. The metabolic pathway and enzymes involved in the key steps of this pathway are represented in Figure 2 and a brief summary of the metabolic pathways involved in the microbial fumaric acid production is provided in reviews by Engel et al. [3] and $\mathrm{Xu}$ et al. [5]. Additionally, it was identified that amino acid and fatty acid metabolism, as well as activation of the glyoxylate pathway, can have a potential impact on FA accumulation [25-27].

\section{Rhizopus sp. mediated fumaric acid production}

Fungal species have been employed to produce a vast array of compounds and consumables. Multicellular filamentous fungi or molds are extensively used to produce fermented foods, secondary metabolites, and industrial enzymes. Rhizopus sp. is an example of filamentous fungi, belonging to the "Mucoraceae" family, that is used to produce a wide array compounds ranging from fermented food products to platform chemicals such as fumaric acid $[20,28,29]$.

Rhizopus oryzae, generally regarded as safe (GRAS), has usually been employed for FA production via fermentation at neutral $\mathrm{pH}$ values to produce fumarate salts [22]. This fungal species is subdivided into two groups (Type I and Type II) according to their genetic and phenotypic differences. Type I accumulates lactic acid whereas Type II mostly synthesize FA in the presence of fermentable sugars. The main difference between these two types is that Type I contains two lactate dehydrogenase $(I d h)$ genes, IdhA, and $I d h \mathrm{~B}$, whereas Type II only has one of the genes IdhB. The lactate dehydrogenase enzyme, LdhA, encoded by the IdhA gene enables the conversion of pyruvic acid to lactic acid whereas the enzyme encoded by $I d h B$ gene possesses insufficient reductive capacity. This reduced capacity allows for the conversion of excess pyruvate to fumaric acid and/or ethanol [28,30]. Table 1 provides a summary of FA production studies conducted using $R$. oryzae. 
Table 1. Summary of fumaric acid production studies carried out using $R$. oryzae.

\begin{tabular}{|c|c|c|c|c|}
\hline Carbon source & Fermenter & $\begin{array}{l}\text { Fumaric acid } \\
\text { concentration }\end{array}$ & $\begin{array}{l}\text { Productivity } \\
(\mathrm{g} / \mathrm{L} / \mathrm{h})\end{array}$ & Reference \\
\hline Glucose & Stirred tank & $56.2 \mathrm{~g} / \mathrm{L}$ & 0.7 & [31] \\
\hline Glucose & Stirred tank & $41.1 \mathrm{~g} / \mathrm{L}$ & 0.37 & [32] \\
\hline Glucose & Stirred tank & $32.1 \mathrm{~g} / \mathrm{L}$ & 0.32 & [33] \\
\hline Glucose & Stirred tank & $30.2 \mathrm{~g} / \mathrm{L}$ & 0.19 & {$[22]$} \\
\hline Xylose & Shake flask & $28.4 \mathrm{~g} / \mathrm{L}$ & - & [34] \\
\hline Xylose & Shake flask (immobilization) & $45.3 \mathrm{~g} / \mathrm{L}$ & - & [35] \\
\hline Glucose/xylose & Shake flask & $46.7 \mathrm{~g} / \mathrm{L}$ & - & [36] \\
\hline Corn straw & Shake Flask & $27.8 \mathrm{~g} / \mathrm{L}$ & 0.33 & [37] \\
\hline Cornstarch & Shake flask (Simultaneous saccharification fermentation) & $44.1 \mathrm{~g} / \mathrm{L}$ & 0.53 & [38] \\
\hline Cornstarch & Shake Flask & $45.0 \mathrm{~g} / \mathrm{L}$ & 0.55 & [32] \\
\hline Brewery wastewater & Shake Flask & $31.3 \mathrm{~g} / \mathrm{L}$ & - & [39] \\
\hline Apple juice extraction waste & Shake Flask & $25.2 \mathrm{~g} / \mathrm{L}$ & 0.35 & [40] \\
\hline Diary manure & Stirred tank & $31.0 \mathrm{~g} / \mathrm{L}$ & 0.32 & [41] \\
\hline Apple pomace & Solid state-Rotating drum fermenter & $138 \mathrm{~g} / \mathrm{kg}$ dry weight & - & {$[40]$} \\
\hline Glucose/crude glycerol & Shake flask & $22.81 \mathrm{~g} / \mathrm{L}$ & 0.34 & [42] \\
\hline Synthetic medium & Immobilized fungi & $32.03 \mathrm{~g} / \mathrm{L}$ & 1.33 & [43] \\
\hline Synthetic medium & Immobilized fungi & $40.13 \mathrm{~g} / \mathrm{L}$ & 0.32 & [44] \\
\hline Synthetic medium & Immobilized fungi & $30.3 \mathrm{~g} / \mathrm{L}$ & 0.21 & {$[45]$} \\
\hline Brewery wastewater & Immobilized fungi & $43.67 \mathrm{~g} / \mathrm{L}$ & 1.21 & {$[46]$} \\
\hline Synthetic medium & Immobilized fungi- fed-batch & $85 \mathrm{~g} / \mathrm{L}$ & 4.25 & [47] \\
\hline
\end{tabular}

\section{Limitations of $\mathbf{R}$. oryzae based production}

$R$. oryzae mediated FA production is usually performed at neutral $\mathrm{pH}$ values. The production of the acid during fermentation leads to a significant decrease in $\mathrm{pH}$ from 6 to 4 within the first $48 \mathrm{~h}$ of fermentation. This reduction in $\mathrm{pH}$ causes the rate of production to slow down and eventually cease completely. To prevent this drop in $\mathrm{pH}$, the fumaric acid is converted to fumarate salts by using neutralizers, such as calcium carbonate and sodium carbonate. Fumaric acid is then recovered from the fermentation broth by precipitation, due to its low solubility, following acidification. The requirement of neutralizers and acids during the recovery step leads to high consumption of bases and acids as along with the production of large quantities of salts. Calcium carbonate is commonly used as the neutralizer but the insolubility of calcium salts causes considerable power consumption and process difficulties, such as increased viscosity and associated power consumption $[22,39,48]$. Hence, sodium carbonate is mainly proposed as a neutralizing agent because the sodium salt of fumarate is soluble. Gangl et al. [48] have performed a comprehensive comparison of the advantages and disadvantages of using calcium carbonate and sodium carbonate as the neutralizing agent.

Some of the above-mentioned limitations of the fungal strain mediated fumaric acid fermentation can be overcome by performing the fermentation at a lower $\mathrm{pH}$. The move to a lower $\mathrm{pH}$ fermentation reduces the quantity of neutralizing agent required in order to maintain $\mathrm{pH}$ close to neutral as well as the quantity of salt generated during the recovery of fumaric acid from the fermented broth. Moreover, the undissociated fumaric acid so formed can be recovered by the crystallization from the broth due to the low solubility of FA. Additionally, the direct recovery of fumaric acid from the broth using adsorbents, such as polyvinyl pyridine (PVP) resin and Amberlite IRA900 , would be possible [5]. This strategy of FA recovery using adsorbents was successfully used by Cao et al. [47] to recover high purity FA following desorption and acidification. To this effect, the shortened duration of $\mathrm{pH}$ control and fermentation at $\mathrm{pH} 5$ was proposed [22]. It was observed that eliminating off $\mathrm{pH}$ control at $90 \mathrm{~h}$ instead of $120 \mathrm{~h}$ did not lead to important effects on FA production. The drawback of this strategy is that $\mathrm{pH}$ control is required during the initial stages of fermentation even though reduced consumption of the neutralizing agent is achieved.

Moreover, it has been observed that the morphology of the fungus during fermentation has a profound impact on FA production and has been extensively studied by Engel et al. [22] and Zhou et al [49]. It was also observed by them that the fungal pellet diameter played a key role in FA productivity. Zhou et al. [49] concluded that lower pellet diameter enhanced for mass transfer as well as better contact with more of the actively growing fungi present on the surface of the culture. Furthermore, it was observed by Engel et al. [22] that an anaerobic environment was present at the core of the fungal pellet. Therefore, fungal pellets of smaller diameter are preferred which requires the optimization of conditions, such as agitation rate, $\mathrm{pH}$ conditions, temperature, and working volume, to achieve the optimum pellet diameter. This dependence on fungal morphology further adds to the complexities associated with 
fungal bioproduction of fumaric acid. To this effect, immobilization of fungi on the support have been proposed to reduce the effects of pellet morphology and has been found effective in increasing FA productivity as well as its yield $[43,46,47]$. Reduction in the duration of fermentation, leading to increased productivity, from $144 \mathrm{~h}$ to $24 \mathrm{~h}$ was observed by Gu et al. [43]. The fumaric acid yield of $32.03 \mathrm{~g} / \mathrm{L}$ was obtained post immobilization of the fungi $R$. arrhizus during this study. In addition, a glucose to nitrogen ratio of the production media has been observed to have a profound impact on FA production. To enable maximum FA yield, nitrogen limitation is recommended to limit fungal growth and achieve optimal diversion of sugars towards fumarate production. It has also been observed that optimal activity of the cytosolic fumarase enzyme was obtained under nitrogen limiting conditions and this activity increased $300 \%$ upon reduction of the urea concentration from 2.0 to $0.1 \mathrm{~g} \mathrm{I}^{-1}[3,19,50]$.

To overcome the above-mentioned complexities of $R$. oryzae mediated fumaric acid production, the genetic transformation of microorganisms, such as S. cerevisiae (capable of growing at low $\mathrm{pH}$ ), to accumulate fumarate has been considered. These strategies of genetic engineering microorganisms to produce fumaric acid are discussed in the following sections.

\section{Improved fumaric acid production through strain improvement and genetic modification}

The lower yield of FA, during bioproduction, compared to chemical synthesis has motivated researchers to investigate strategies for improving the FA yield of the filamentous fungus $R$. oryzae. These strain improvement strategies primarily rely on increasing product yields thereby enhancing the feasibility of commercial bioproduction. These techniques of strain improvement have been extensively reviewed by Meussen et al. [51] and Li et al. [52] but strain improvement techniques targeted towards FA production have so far not been discussed and this discussion occurs in later sections of this review. Moreover, under low pH conditions (3-5), due to reduced fungal growth as well as the complexities of this fermentative fungal strain mediated fumaric acid production has led researchers to investigate the possibility of using alternate microorganisms for the production of FA. Developments in genetic engineering techniques, such as genetic transformation and overexpression of homologous genes, such as fum, pyc and $m d h$, and metabolic engineering, have provided the tools for improving microbial production of organic acids, such as: malic acid, succinic acid, and fumaric acid [29,51].

\section{Strain improvement strategies for $\mathbf{R}$. oryzae}

\section{Random mutagenesis}

Random mutagenesis involves the use of chemical mutagens, such as diethyl sulfate, nitrosoguanidine and diethyl sulfate, or radiation, such as UV light and gamma radiation, to induce changes in the genetic makeup of an organism. This technique has for centuries been employed for the development of novel strains. The main drawback of this technique is that following induction of the mutation, multiple mutant strains are generated. These strains generate the need to be screened and a suitable strain selected which is time-consuming and laborious [19,51]. The mutant strains selected were able to produce higher yields of fumaric acid than the parent strain but these titers were significantly lower than the maximum titers reported for strains obtained from culture collections (represented in Table 2) but comparable to other titers that have been reported [31-33]. Hence, random mutagenesis can be used as a viable tool for strain improvement. The adoption of novel strategies, discussed in a later section, in combination with random mutagenesis can be useful for the selection of appropriate high yielding strains. Moreover, the use of this technique generates multiple mutations in the

Table 2. Random mutagenesis of $R$. oryzae and fumaric acid concentrations obtained (R. oryzae reassigned as $R$. arrhizus, [53]).

\begin{tabular}{lcccc}
\hline Fungal strain & Mutagen & Fumaric acid titer $(\mathrm{g} / \mathrm{L})$ & Yield $(\mathrm{g} / \mathrm{g})$ & Reference \\
\hline Pure culture & - & & & \\
$\quad$ R. arrhizus NRRL 2582 & - & 103 & 0.79 & {$[54]$} \\
$\quad$ arrhizus NRRL1526 & & 98 & 0.82 & {$[55]$} \\
Mutant & & 32.1 & & \\
R. oryzae RUR709 & UV and $\gamma$-ray mutagenesis & 57.4 & 0.45 & {$[33]$} \\
R. oryzae ZD-35 & UV irradiation & 52.7 & 0.67 & {$[32]$} \\
R. oryzae ME-F01 & UV and nitrosoguanidine & 44.1 & - & {$[56]$} \\
R. oryzae ME-F12 & Nitrogen ion implantation & 49.4 & 0.44 & {$[38]$} \\
R. oryzae FM19 & Femtosecond laser irradiation & 0.56 & {$[25]$} \\
\hline
\end{tabular}


microorganism and the other metabolic activities may be hampered in addition to the targeted metabolite production. Also, the damage to the DNA by the use of high energy radiations may prevent DNA replication and hamper the overall transfer of the desired trait.

\section{Homologous and heterologous gene expression in R. oryzae for fumaric acid production}

Fumaric acid is an intermediate of the TCA cycle, as mentioned in the earlier section, but the fumaric acid generated during the oxidative pathway is not accumulated as it is consumed for cell growth and maintenance. The accumulation of fumaric acid occurs via the reductive TCA pathway, as observed in the case of $R$. oryzae. Multiple enzymes are involved in the synthesis of fumaric acid from pyruvate. Pyruvate carboxylase (PYC) catalyzes the ATPdependent condensation of pyruvate and $\mathrm{CO}_{2}$ to form oxaloacetic acid (OAA). The OAA, so formed, is converted to malate by malate dehydrogenase (MDH), followed by conversion of malate to fumarate by the enzyme fumarase. [57] The overexpression of the enzymes involved should be able to increase fumarate production.

Zhang et al. [57] observed a $26 \%$ increase in fumaric acid production in their study by transforming $R$. oryzae to express homologous and heterologous enzymes. They introduced the exogenous phosphoenolpyruvate carboxylase enzyme (PEPC) in addition to PYC using the plasmid (pPyrF2.1A) mediated transformation of fungi $R$. oryzae M16. The PEPC enzyme, not present in $R$. oryzae, is involved in the conversion of phosphoenolpyruvate to OAA with $\mathrm{CO}_{2}$ fixation. Increased PYC and PEPC activity of $3-6 \mathrm{mU} / \mathrm{mg}$ were observed. The redirection of this metabolic pathway resulted in the introduction of these genes that lead to an increase in fumarate production [57]. In another study, the impact of the overexpression of the fum $R$ gene that encodes for the fumarase enzyme was investigated. It was observed that the over-expression of fumarase in $R$. oryzae lead to malic acid production instead of FA. The over-expressed fumarase is also able to catalyze the hydration of FA to malic acid. Hence, it can be concluded that the presence of fumarase alone may not be responsible for FA accumulation during $R$. oryzae mediated fermentation [58]. To this effect, the observation by Peleg et al. has a particular significance [59]. They identified two isoenzymes of fumarase with a different localization within the cell. One isoenzyme was solely present in the cytosol whereas the other was found in both the cytosol and in the mitochondria. It was also observed that the inhibition of fumarase activity by cycloheximide only affected fumaric acid production and decreased the activity of the cytosolic fumarase isoenzyme [59]. Therefore, the transformation of $R$. oryzae with the appropriate isoenzyme has the potential to improve FA production as the reductive TCA cycle occurs in the cytosol.

In addition, a dicarboxylic acid transporter with high selectivity for fumaric acid can also play an important role in its production with $R$. oryzae. This conclusion is supported by the observation that malic acid production by Aspergillus oryzae NRRL 3488 was improved twofold by the overexpression of a C4-dicarboxylate transporter. The overexpression of malate dehydrogenase and pyruvate carboxylase in conjunction with the transporter led to an additional increase of $27 \%$ and achieved a malic acid concentration of $154 \mathrm{~g} / \mathrm{L}$ in $164 \mathrm{~h} \mathrm{[60].} \mathrm{The} \mathrm{high} \mathrm{concentration} \mathrm{of} \mathrm{malic}$ acid achieved in this study points to the possibility of achieving a similar titer for fumaric acid by providing an efficient system for the conversion of the malic acid into fumaric acid.

\section{Fumaric acid production in recombinant microorganisms}

The limitations of $R$. oryzae mediated synthesis of fumaric acid, discussed previously in Limitations of $R$. oryzae based production section, has motivated researchers to investigate the possibility of using other microorganisms, particularly Escherichia coli and Saccharomyces cerevisiae, to produce fumaric acid. To this effect, the metabolic pathways that influence FA production have to be investigated prior to the generation of recombinant strains. Cellular metabolism is highly regulated and made up of a plethora of various components, such as transcripts, proteins, and metabolites, and the production of the compound of interest is directly linked to the cellular activities. Hence, an accurate understanding of the metabolic states and regulatory mechanisms are important.

The most effective way to evaluate the metabolic state is to quantify various metabolites. Therefore, the advances in analytical techniques, such as NMR, gas chromatography-mass spectrometry (GC-MS), gas chromatography time-of-flight mass spectrometry (GCTOF) and liquid chromatography-mass spectrometry (LC-MS), has enabled high-throughput analysis of the metabolites. This has allowed for quick comparative analysis of metabolite profiles post-genetic modification and provides an understanding of the physiological state of the cell, which in turn provides us 
with an understanding of their metabolic status. To further improve an understanding of the metabolic state of the cell, isotopomer experiments can be performed. These studies provide us with additional information on intracellular fluxes and metabolic rates. ${ }^{13} \mathrm{C}$-labeled glucose is the most frequently used isotopomer. The monitoring of the distribution of the isotopomer, as the labeled glucose is metabolized, can be used to estimate metabolic reaction rates. Hence, provide quantitative information regarding carbon flow through the metabolic pathways [60,61]. An understanding of this would allow for the selection of the metabolic pathway of interest that would enable maximum conversion of glucose to fumaric acid with reduced byproduct formation.

Improvement of the cellular systems by metabolic engineering strategies is vital to enhance the overall productivity and yield of the target compound. The development of stoichiometric metabolic models which includes all enzymatic reactions of organisms, such as $E$. coli and S. cerevisiae, has enabled the prediction of metabolic states using in silico simulations. Metabolic flux analysis (MFA), flux balance analysis (FBA), and metabolic pathway analysis (MPA) are the most popular tools in stoichiometric metabolic network analysis. Additionally, database resources, such as UniProt, BRENDA, BioCyc, KEGG, and the Enzyme Commission database, can be used for the reconstruction of a metabolic pathway $[29,62]$. The above-mentioned tools for metabolic engineering have been extensively used for transformation of $E$. coli and $S$. cerevisiae strains but have not been readily used for the transformation of $R$. oryzae. There are only a few reports the transformation of $R$. oryzae that adopted metabolic flux analysis for metabolic engineering of considering this fungal strain.

One such early study used TELUX, a specific radioactivity curve-matching program, to investigate glucose metabolism by $R$. oryzae. In the study, ${ }^{14} \mathrm{C}$ isoprotomer labeled glucose was used to understand glucose metabolism and thereby increase lactate yield. This was followed by UV-mediated random mutagenesis and the selection of a lactate overproducing strain. The prediction of metabolic pathway modification that led to lactate overproduction, provided by glucose flux analysis, helped in the identification of a suitable strain. This selected strain showed reduced ethanol production and chitin synthesis, as predicted, and a lactic acid yield of $30 \mathrm{~g} / \mathrm{L}$ was obtained [63]. In another study, the results of the carbon flux analysis of a mutant lactic acid overproducing strain, $R$. oryzae R1021, and the parent strain, $R$. oryzae R3017, showed that pyruvate was the key metabolic intermediate. The mutant strain showed reduced loss of carbon to ethanol and acetyl-CoA and provided a lactic acid yield of $79.4 \mathrm{~g} / \mathrm{L}$, which was $52 \%$ higher than that of the parent strain [64]. These studies focused on the synthesis of lactic acid but the understandings can possibly be adopted for FA production. Such a study on metabolic profiling, post femtosecond laser-mediated random mutagenesis, identified that modifications to amino acid and fatty acid metabolism led to a 1.59-fold increase in FA production [25]. These studies used MFA or metabolic profiling for understanding carbon metabolism either before or after random mutagenesis but not for designing metabolic pathways and transforming the fungi. The importance of a better understanding of metabolic activities using metabolite profiling is further signified by the observation that the FA production was enhanced by $39.7 \%$ as a result of supplementation with $1 \%$ linoleic acid during $R$. oryzae mediated fermentation at low $\mathrm{pH}(\mathrm{pH} 3)$ condition. The strategy of supplementation with linoleic acid was adopted based on the understanding of the changes to the metabolic activity at low pH conditions provided by GC-MS assisted metabolite profiling [65]. The use of in silico metabolic modeling was found to be effective in designing a metabolic engineering strategy to achieve a higher FA titer in T. glabrata [66]. Hence, an in silico model of fumarate synthesis of $R$. oryzae is essential to achieve effective conversion of glucose to fumarate.

The successful adoption of MFA assisted strain selection, in combination with strain improvement strategies, can also be adopted to improve FA yield in $R$. oryzae. MFA can act as an important tool for the selection of an appropriate high FA producing strain following random mutagenesis. Moreover, it would be possible to obtain far higher titers for FA by using MFA mediated design and then transform the fungal strain using targeted genetic modification approaches. These strategies would enable reduced byproduct synthesis, such as ethanol and malic acid, as well as effective redirection of a metabolic pathway that allows for maximum productivity by optimal conversion of glucose to $\mathrm{FA}$, as observed in case of both $E$. coli and S. cerevisiae discussed below.

\section{Escherichia coli}

High concentrations of fumaric acid are not normally produced in E. coli and $20 \mathrm{~g} / \mathrm{L}$ has been shown to have an impact on cell growth [26]. The adoption of genetic engineering strategies has allowed the use of $E$. coli for the production of a diverse range of 
compounds and possibly fumarate. This possibility has been investigated. The de-repression of the glyoxylate pathway in combination with overexpression and deletion of specific genes was exploited to induce FA accumulation [26,27]. Song et al. [26] used rational metabolic engineering strategies coupled with in silico flux response simulation to develop a novel $E$. coli strain. The in silico flux modeling simulation ( $E$. coli genome-scale metabolic model EcoMBEL979) was used to investigate the influence of PEPC flux on fumarate production and helps to understand the response to a metabolic shift. It was observed the overexpression of PEPC was able to increase the FA production and further genetic modifications selected based on their effect on FA production. The final concentration of FA was achieved by transforming $E$. coli to overproduce FA via the noncyclic glyoxylate route. The isocitrate lyase repressor gene ic/R was deleted to enhance the carbon flow into the glyoxylate shunt in addition to the silencing of fumarase isozymes. Moreover, endogenous PEPC was also overexpressed to increase the reductive TCA cycle flux to achieve FA production. Additionally, genes involved in the further conversion of FA and byproduct syntheses, such as $\operatorname{arc} \mathrm{A}$, aspA, lacL, and ptsG, were deleted. FA production with the final strain obtained was $28.2 \mathrm{~g} / \mathrm{L}$, and a productivity of $0.448 \mathrm{~g} / \mathrm{L} / \mathrm{h}$ under aerobic fed-batch culture conditions [26].

The glyoxylate pathway flux was enhanced similarly to obtain a genetically modified strain of $E$. coli capable of utilizing glycerol as a feedstock for FA production. The conversion of fumaric acid to malic acid was prevented by the deletion of fumA, fumB, and fumC genes that code for three fumarases. It was observed that the overexpression of glyoxylate shunt and PEPC enzyme led to enhanced fumaric acid production as well as reduction of acetate formation (a byproduct). Additionally, the gene coding for aspartase, which converts fumarate to aspartate, was deleted to prevent the reverse conversion. An FA concentration of $41.5 \mathrm{~g} / \mathrm{L}$ was achieved with the overall productivity of $0.51 \mathrm{~g} / \mathrm{L} / \mathrm{h}$ following fermentation under fed-batch conditions. This corresponded to $70 \%$ of the maximum theoretical yield [27]. The concentration of FA achieved during these investigations is still lower than the titers obtained by culturing $R$. oryzae. These studies focused primarily on the redirection of the TCA cycle towards the glyoxylate pathway for FA accumulation. However, the reductive TCA pathway has the potential to produce 2 moles of FA for every mole of glucose. This yield is double than that of the theoretical maximum of the oxidative glyoxylate pathway $(1 \mathrm{~mol} / \mathrm{mol}$ glucose) [67]. Hence, it may be possible to obtain even a higher titer for FA with the enhancement of the reductive TCA pathway along with the genetic modification strategies adopted during these studies.

Another metabolic pathway that has been investigated for fumarate bioproduction in $E$. coli was the introduction of the mammalian urea cycle [68]. Fumaric acid is produced as a byproduct in this metabolic pathway during the conversion of L-arginosuccinate to arginine. The recombinant E. coli (ABCDIARAC) was produced by blocking the Krebs cycle and introducing the urea cycle. To improve FA production, several genes, such as fumA, fumB, fumC, frdABCD, $i c / \mathrm{R}$, and $\operatorname{arc} \mathrm{A}$, were deleted. Additionally, the urea cycle was introduced by overexpressing the homologous carAB, argl, and heterologous rocF genes which encoded for carbamoyl phosphate synthase (CPS), ornithine carbamoyl transferase (OTC), and arginase, respectively. The strain isolated was able to yield a fumarate titer of $11.38 \mathrm{mmol} / \mathrm{L}(1.32 \mathrm{~g} / \mathrm{L})$ and moreover, it was concluded that cell growth and the derepression of the Krebs cycle were required for hightiter FA production in aerobic conditions [68]. In another study by the same research group, the FA yield of the strain was further improved to $9.42 \mathrm{~g} / \mathrm{L}$ by the overexpression of the homologous C-4 dicarboxylate carrier gene $d c u B$ [69]. These studies have failed to achieve the FA yields reported for $R$. oryzae but shows that targeted multilevel genetic transformations are essential to improve fumarate yield.

\section{Saccharomyces cerevisiae}

The yeast, S. cerevisiae is a well-established industrial production organism and it possesses exceptional cultivation characteristics. They require only simple media for growth and are tolerant to the presence of inhibitors. Moreover, there is a high degree of tolerance to (high) sugar concentrations and their ability to grow under acidic conditions. The inability to grow under low $\mathrm{pH}$ conditions is one of the main limitations to $R$. oryzae mediated FA production. S. cerevisiae is not naturally capable of accumulating FA and the feasibility of the induction of FA accumulation by engineering $S$. cerevisiae has been investigated [5].

The introduction of heterologous cytosolic $R$. oryzae malate dehydrogenase, high-level expression of the $R$. oryzae fumarase and overexpression of the native cytosolic pyruvate carboxylase was observed to only yield an FA concentration of $3.2 \mathrm{~g} / \mathrm{L}$ [70]. In a similar study, simultaneous co-expression of both reductive and oxidative routes of FA was investigated. This 
co-expression of heterologous RoMDH and RoFUM1 failed to accumulate $F A$ in the engineered strain. It was also observed that the fumarase deleted strain could produce FA via the oxidative route. Moreover, it was observed that the introduction of the heterologous reductive route of FA led to lower production of FA but the introduction of a heterologous gene for pyruvate carboxylase (RoPYC) led to a higher FA titer $(2.48 \mathrm{~g} / \mathrm{L})$. The maximum FA titer obtained, under optimal conditions of nitrogen limitation and the presence of biotin (co-factor of PYC), was $5.6 \mathrm{~g} / \mathrm{L}$ [5].

Another strategy investigated improved FA yield, investigated by the same group, was to subject the $S$. cerevisiae CEN.PK2-1C pdc1adh1fum1 strain to sitedirected mutagenesis for improving the catalytic activity of RoPYC as well as overexpression of the same strain. The site-directed mutagenesis was able to improve the catalytic activity of it by reducing the conformational instability afforded by the presence of the amino acid proline at 474 positions. Substitution of the amino acid with asparagine led to improved FA production than the wild type strains. The best FA yield of $448.4 \mathrm{mg} / \mathrm{L}$ was obtained following biotin supplementation of $56 \mu \mathrm{g} / \mathrm{L}$ [71]. This yield is considerably lower than their previous study which yielded $5.6 \mathrm{~g} / \mathrm{L}$ fumarate [5]. Hence, a better understanding of the underlying mechanism of the metabolic pathways influencing fumarate production is essential for successful genetic modification strategies to improve its yield.

\section{Torulopsis glabrata}

Recently, multivitamin auxotrophic mutant strains of T. glabrata have been investigated for fumaric acid production following genetic modifications. The auxotrophic strains have been known to produce high titers of pyruvic acid and reported to have a higher glucose and acid tolerance than S. cerevisiae [66]. Hence, is an ideal strain for use in the industrial production of organic acids, mainly pyruvate, but has been subjected to genetic modification to enable the production of malic acid as well as FA [66,72].

The genetic modification strategy adopted involved identification of the metabolic pathways that contributed to the accumulation of FA in the cytosol followed by the introduction of enzymes involved in the metabolic pathways. To this effect, key enzymes affecting fumarate accumulation, involved in both carbon and nitrogen metabolism such as argininosuccinate lyase (ASL), adenylosuccinate lyase (ADSL), fumarylacetoacetase (FAA), and fumarase (FUM1), were identified using in the silico metabolic model (iNX804). The overexpression of the enzymes individually led to the accumulation of FA in the modified strains compared to the parent auxotrophic T. glabrata strain but ASL and ADSL were identified as the key enzymes leading to higher FA accumulation. Optimization of overexpression levels of enzymes ASL and ADSL gave a FA titer of $5.62 \mathrm{~g} / \mathrm{L}$. This was further improved by introducing a heterogeneous gene, SPMAE1, that encodes for a C4-dicarboxylic acids transporter in S. pombe. The final titer obtained was $8.83 \mathrm{~g} / \mathrm{L}$ [66].

Genetic modification of T. glabrata was performed in another study which involved the introduction of heterogenous $R$. oryzae genes that code for the fumarase enzyme (RoFUM), pyruvate carboxylase (RoPYC) and malate dehydrogenase (RoMDH) as well as the C4dicarboxylic transporter, SpMAE1, from S. pombe. The binding site $B$ of RoFUM was subject to site-directed mutagenesis to enhance the catalytic efficiency of the enzyme. The resulting modified strain yielded a fumarate titer of $5.1 \mathrm{~g} / \mathrm{L}$. Further increases in FA yield were achieved by subjecting the strain to additional modification. This additional modification involved deletion of the gene, ade12, that codes for adenylosuccinate synthetase that is involved in purine metabolism. The obtained strain resulted in a significantly higher FA yield of $9.2 \mathrm{~g} / \mathrm{L}$ as well as increased acid tolerance [72].

\section{Limitations of current genetic engineering strategies}

The concentrations of the fumaric acid obtained using genetically engineered strains of $E$. coli, S. cerevisiae, and $T$. glabrata are significantly lower than that reported for $R$. oryzae, this is summarized in Table 3 . Despite the lower yields, genetic modification techniques are an important tool to improve to further improve fumarate yield. These genetic and metabolic engineering strategies exhibit a special significance to decrease the cost of production as well as efficient utilization of biomass and industrial residues as feedstocks for fumaric acid production. Moreover, these modifications allow for the alleviation of the problems associated with $R$. oryzae mediated FA bioproduction discussed in Limitations of $R$. oryzae based production section. Advanced genetic and metabolic engineering strategies that may be adopted for strain improvement are discussed in the following sections.

\section{Advanced genetic and metabolic engineering strategies for fumaric acid production and its derivatives}

Advances in genetic and metabolic engineering have permitted for modifications to inherent metabolic 
Table 3. Comparison of fumaric acid titer and yield of natural strains and genetically engineered strains.

\begin{tabular}{|c|c|c|c|}
\hline Strains & Fumaric acid titer ( $\mathrm{g} / \mathrm{L}$ ) & Yield $(\mathrm{g} / \mathrm{g})$ & Reference \\
\hline \multicolumn{4}{|l|}{ Natural strains } \\
\hline \multirow[t]{2}{*}{ R. arrhizus } & 103 & 0.79 & [54] \\
\hline & 98 & 0.82 & [55] \\
\hline \multirow{3}{*}{ R. oryzae } & 56.2 & 0.54 & [31] \\
\hline & 56.5 & 0.94 & [73] \\
\hline & 40.5 & 0.51 & [74] \\
\hline \multicolumn{4}{|l|}{ Engineered strains } \\
\hline E. coli CWF812 & 28.2 & 0.39 & [26] \\
\hline E. coli $\mathrm{EF} 02(\mathrm{pSCppc})$ & 41.5 & 0.44 & [27] \\
\hline R. oryzae ppc & 25 & 0.78 & [57] \\
\hline S. cerevisiae FMME 001 PYC2 + RoMDH & 3.18 & 0.05 & [70] \\
\hline S. cerevisiae FMME $006 \Delta \mathrm{FUM} 1+\mathrm{RoPYC}+\mathrm{RoMDH}+$ RoFUM1 & 5.64 & 0.11 & [5] \\
\hline T. glabrata $\mathrm{ASL}_{(\mathrm{H})}-\mathrm{ADSL}_{(\mathrm{L})}-\mathrm{SpMAE1}$ & 8.83 & 0.15 & [66] \\
\hline T. glabrata ( $\Delta$ ade12) - PMS - P160A & 9.2 & 0.15 & [72] \\
\hline
\end{tabular}

pathways to be carried out as well as the expression of relevant heterologous pathways in desired microorganisms. Few of such strategies that can be considered for the production of fumaric acid as well as its derivative esters are discussed in the following sections.

\section{Genetic and metabolic engineering tools for the bioproduction of fumaric acid}

Genetic transformation systems have allowed for the introduction of foreign DNA in filamentous fungi, such as $R$. oryzae, to produce strains with desired characteristics. Most of these transformation techniques, such as plasmid vector-mediated transformation, produce strains with the transforming DNA remaining extrachromosomally leading to low mitotic stability [75]. Moreover, alternate transformation techniques, such as protoplast (spheroplast) fusion, are complicated as well as inconsistent due to inherent variability in the composition of fungal cell walls of different strains [52]. To this effect, alternate transformation techniques that provide for stable chromosomal integration of the introduced DNA are required.

\section{Agrobacterium-mediated transformation (AMT)}

For successful genetic transformation, an efficient transformation technique is required to insert genes of interest. Agrobacterium tumefaciens-mediated transformation (ATMT) is a such technique and has been used for the transformation of filamentous fungi. The main advantage of this method is that it generates transformants that have single genomic integration of the desired gene [76]. The soil bacterium A. tumefaciens has the ability to transfer a part of its DNA called transfer DNA (T-DNA) and this ability is exploited for heterologous protein expression in the host organism. For this purpose, the T-DNA is altered to contain the gene of interest. After the introduction of the modified T-DNA into the host by $A$. tumefaciens, the T-DNA integrates into the chromosome of the host, thereby achieving the transformation of the host organism. A brief summary of the technique and factors affecting AMT efficiency has been provided by Li et al. [52].

The filamentous fungus $R$. oryzae has been subjected to transformation using the AMT technique [75]. It was concluded that the technique was not suitable for the expression of the gene of interest in $R$. oryzae due to the presence of a possible defense mechanism. However, more recent researches have proved that heterologous gene expression is possible. The ectomycorrhizal fungus Laccaria laccata was successfully transformed through the ATM technique to express hygromycin B phosphotransferase (hph) [76]. In another study, the filamentous fungus Trichoderma reesei QM9414 was transformed using the AMT technique which resulted in the formation of a hygromycin B-resistant fungi [77]. On the other hand, a decrease in resistance to hygromycin $B$ was observed during several cultivation cycles of the fungus Backusella lamprospora post successful integration of the heterologous gene [78].

The AMT technique has been used for the genetic engineering of a diverse group of organisms but there are no reports of using this technique for the transformation of fungi for organic acid production, especially fumaric acid. The requirement of binary vectors for this method may be one of the probable reasons. These binary vector systems are tedious to prepare [79]. Also, there are numerous factors that play a key role in determining the transformation efficiency which needs to be optimized. This genetic modification and successful integration is a time-consuming process. Moreover, the presence of a possible defense mechanism against the presence of heterologous genes may have contributed to the non-adoption of the AMT technique for the fungal transformation [75]. 
The loss of introduced heterologous characteristics with time further contributes to the unsuitability of the technique [78].

\section{crispr cas-9}

The advances in genetic engineering technologies have created a number of opportunities for basic biological research with filamentous fungi. Clustered regularly interspaced short palindromic repeats (CRISPR)-CRISPRassociated proteins (Cas) has recently emerged as a potent genetic engineering technique. It is a potential candidate to resolve the issue of low edit gene frequency observed in the case of filamentous fungi. A comprehensive review of using the system for editing the fungal genome is provided by Deng et al. [80] and Shi et al. [81]. The efficient, simple, and fast technique of genetic modification allows for systemic research on filamentous fungi. This technique is in its infancy and researchers are continuously learning about the potential applications of this potent genome-editing tool.

This genetic engineering technique has been mostly used to perform gene deletion or creating a knockout transformants. The versatility of the Cas9-multiple SgRNA allows for insertion, deletion, and replacement of target genes, thereby generating positive multiple gene modifications. From the fumaric acid production point of view, the system can be used to efficiently produce transformants with the deletion of genes that encode enzymes, such as lactate dehydrogenase and alcohol dehydrogenase, that causes the production of byproducts. This enables the redirection of metabolic flux towards fumarate production [82].

Moreover, this system was used in the development of a genetic engineering toolbox for $A$. niger. The toolbox allows for the generation of strains carrying heterologous expression cassettes at a defined genetic locus. The proof of this concept was shown by the transformation of $A$. niger to produce aconitic acid. The transformed cells produced aconitate only in the presence of the inducer $10 \mu \mathrm{g} / \mathrm{mL}$ doxycycline [83]. Similar expression systems may be used for the production of fumaric acid whereas $R$. oryzae can be transformed to overexpress the enzymes involved in the reductive pathway. Additionally, the enzymes involved in the glyoxylate pathway may be overexpressed leading to the accumulation of succinate and malate which can then be converted to fumaric acid by succinate dehydrogenase and fumarase respectively.

\section{Gene knockout and silencing}

Gene knockout and gene silencing have become important tools for genetic engineering. During gene knockout, the DNA is modified deliberately to remove the entire or part of the gene whereas gene silencing is a natural process where the expression is reduced. The interlinked nature of metabolic pathways, as represented in Figure 3(a), can lead to the production of multiple byproducts in addition to the compound of interest. For example, during the synthesis of fumarate from the glucose, byproducts, such as ethanol, lactic acid, and glycerol, are also synthesized. This leads to the loss or redirection of some of the glucose in the culture towards the synthesis of these byproducts. The inactivation of the genes responsible for the synthesis of the enzymes, or the prevention of their synthesis, involved in the production of these byproducts can lead to the redirection of the metabolic pathway towards the synthesis of only the desired product, as depicted in Figure 3(b).

For example, the inactivation of enzymes pyruvate decarboxylase and lactate dehydrogenase can prevent the formation of the byproducts ethanol and lactate, respectively. This redirects the metabolic pathway towards fumarate synthesis. The inactivation of the enzymes involved can be achieved by the use of techniques such as gene knockout or RNAi (RNA interference) [84]. Gheinani et al. [85] were able to successfully silence the IdhA and IdhB genes of $R$. oryzae with siRNA technology, the most commonly used RNA interference (RNAi) tool and redirects metabolic activity towards ethanol synthesis. Similar deactivation of the lactate dehydrogenase gene of an anaerobic gut fungus, Pecoramyces ruminantium strain C1A was performed and reduced lactate production was observed [86].

\section{Possible metabolic pathway modifications in R. oryzae}

The modification of the metabolic pathway of $E$. coli by activation of the glyoxylate pathway has proved to be successful in enabling the transformed strain to accumulate FA $[26,27]$. Hence, this re-direction of the metabolic pathway in conjunction with the inherent reductive TCA cycle can be introduced in $R$. oryzae to enhance the production of FA. This possibility of transformation has not been reported and the redirected metabolic pathway is represented in Figure 4. This redirection via the glyoxylate pathway has the potential to enhance FA accumulation in this fungal strain. It has been reported that the glyoxylate 
(a)

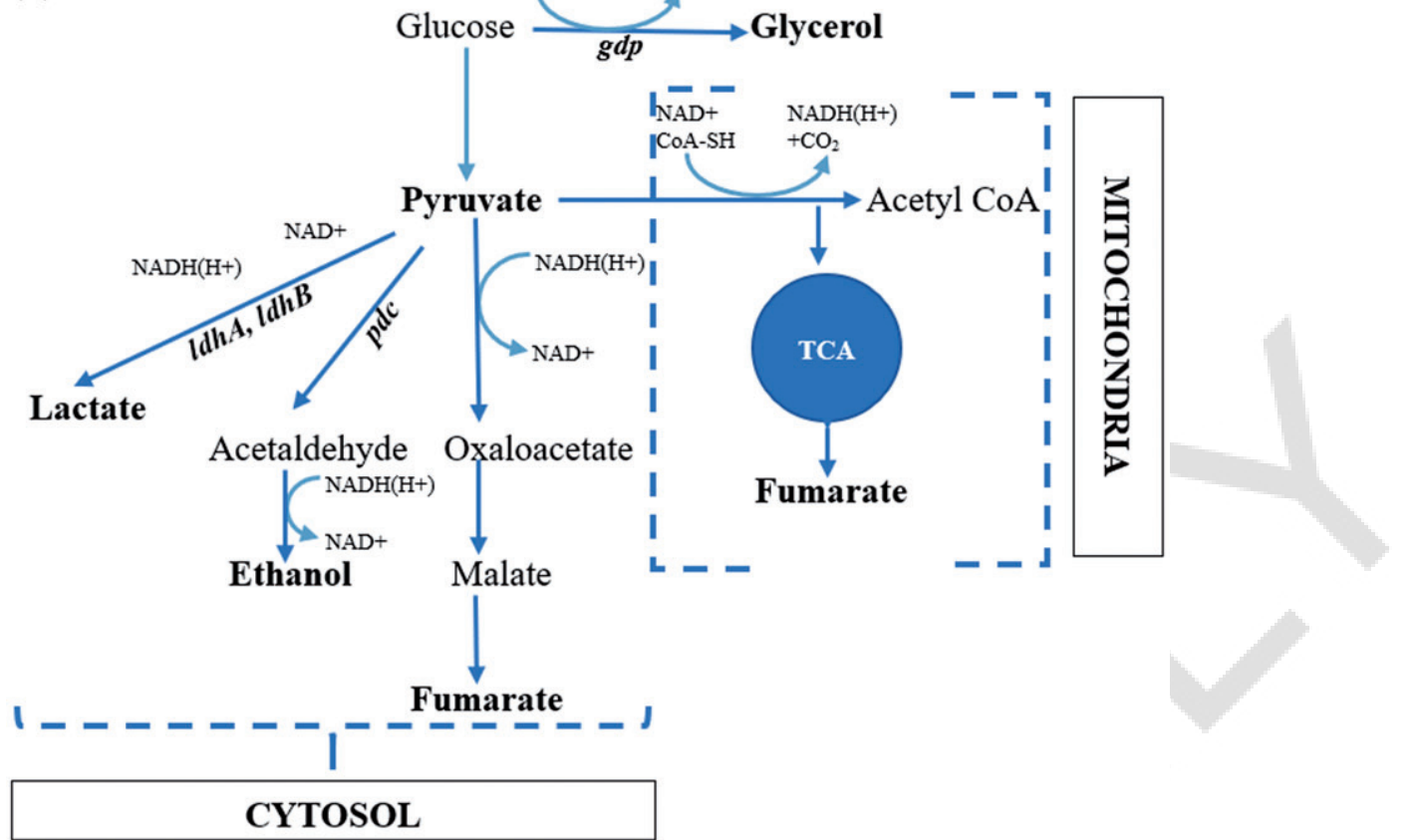

(b)

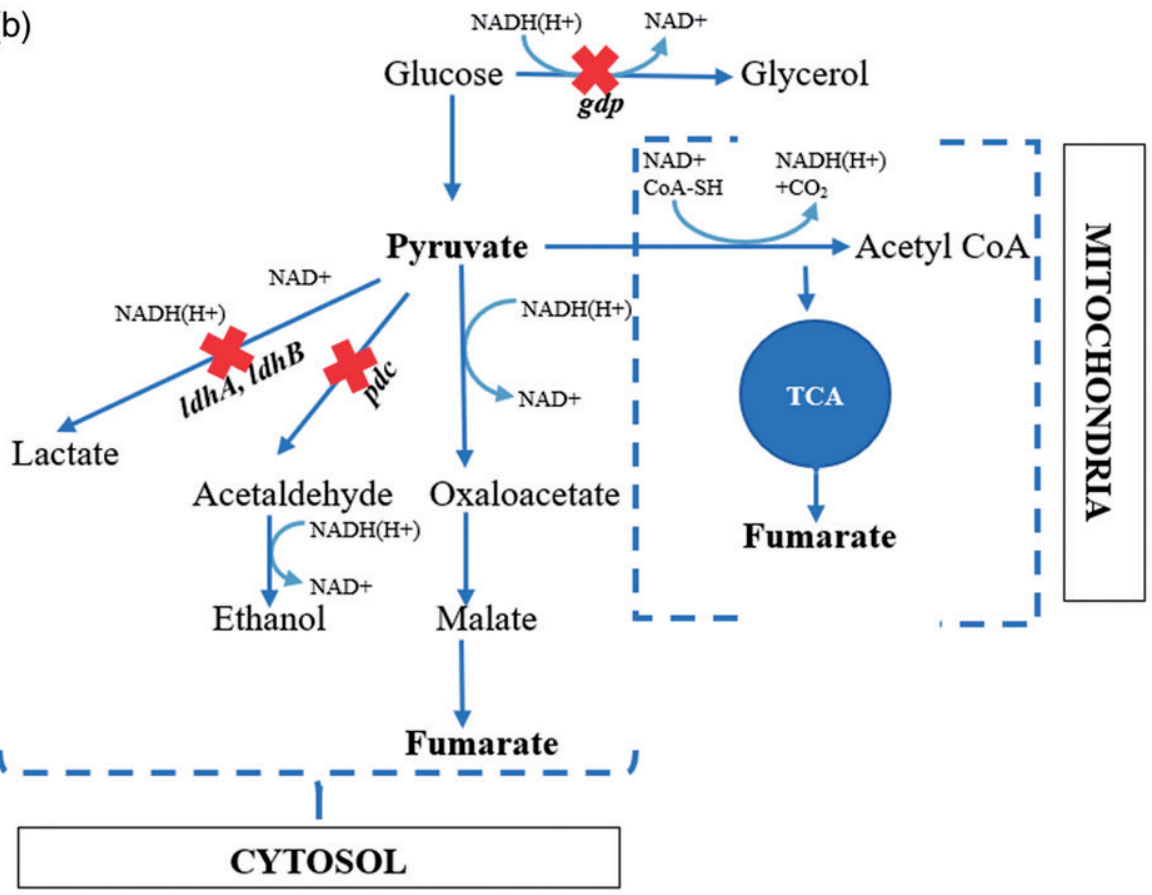

Figure 3. (a) Metabolic pathways involved in the production of fumaric acid and byproducts and (b) reduction of byproduct formation by gene inactivation (represented in red) to obtain a high fumaric acid titer. $g d p$ - Glyceraldehyde-3-phosphate dehydrogenase; Idh-lactate dehydrogenase, and $p d c$ - pyruvate decarboxylase. For color version of the figure refer e-print.

pathway is required for fungal virulence [87]. Hence, a comprehensive understanding of the underlying risk of enhanced fungal virulence, if any, needs to be studied extensively before any commercial application of the transformed strain is considered.

Additionally, metabolic modifications similar to that described by Chen et al. $[66,72]$ can be investigated with $R$. oryzae. These modifications have been able to increase FA titers in T. glabrata. Hence, the introduction of metabolic enzymes involved in nitrogen and purine metabolism are that carbon metabolism can be considered to improve fumarate yield. Moreover, the introduction of an effective C4-dicarboxylic acid transporter, such as SpMAE1 and DcuB, that has been shown to significantly improve fumarate production can be considered [66]. The acid tolerance of 


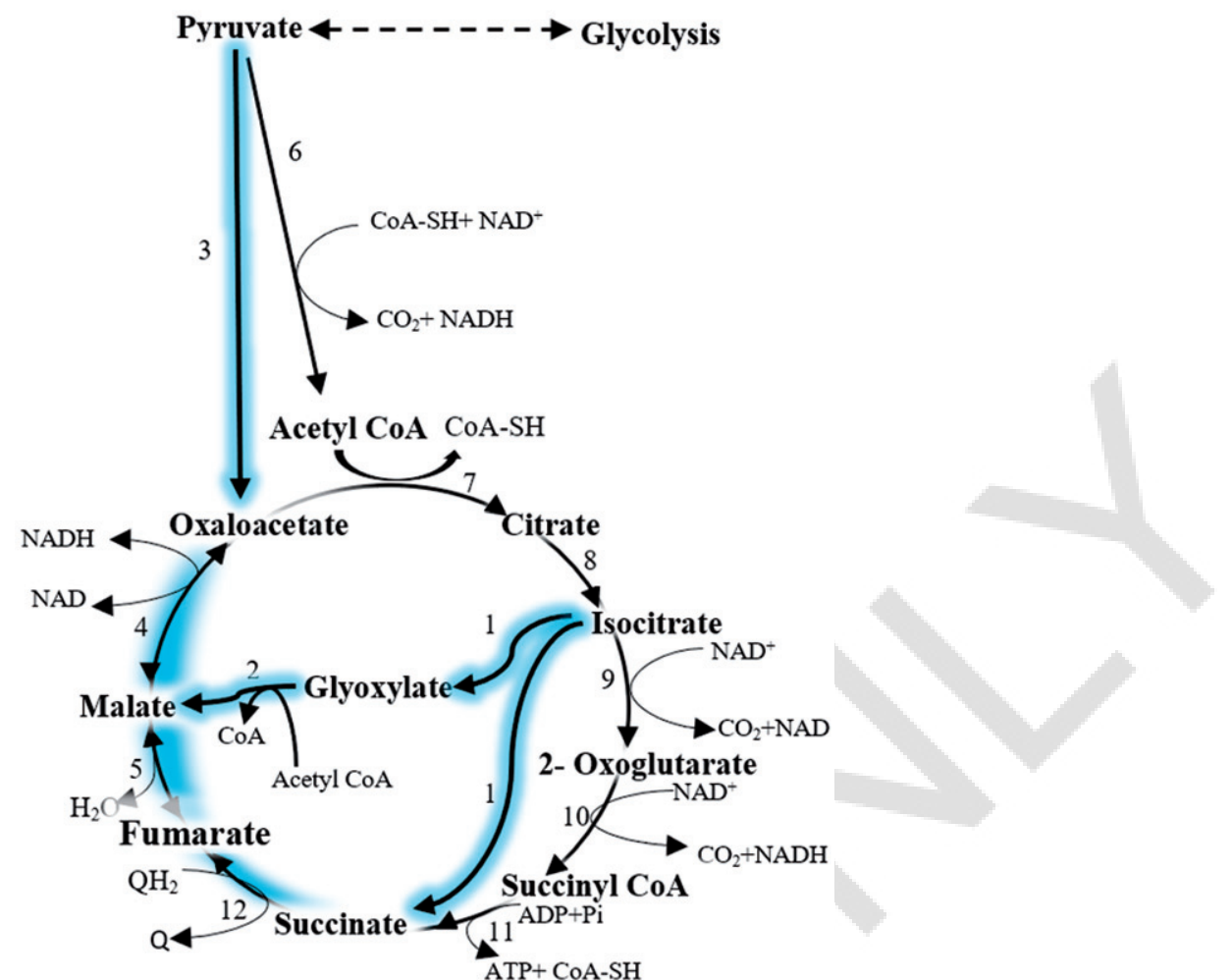

Figure 4. Possible fumaric acid production via the introduction of the glyoxylate pathway (highlighted in blue) in conjunction with reductive TCA pathway (highlighted in blue) in $R$. oryzae. The various enzymes involved in the metabolic pathway have been represented in numbers. 1. Isocitrate lyase (EC 4.1.3.1); 2. malate synthase (EC 2.3.3.9); 3. pyruvate carboxylase (EC 6.4.1.1); 4. malate dehydrogenase ( $E C$ 1.1.1.37); 5. fumarase ( $E C$ 4.2.1.2); 6. pyruvate dehydrogenase $(E C$ 1.2.4.1); 7. citrate synthase (EC 2.3.3.1); 8. aconitase (EC 4.2.1.3); 9. isocitrate dehydrogenase (EC 1.1.1.42); 10. 2-oxoglutarate dehydrogenase (EC 1.2.4.2); 11. succinyl CoA synthase (EC 6.2.1.5); and 12. succinate dehydrogenase (complex III) (EC 1.3.99.1). For color version of the figure refer e-print.

T. glabrata, as well as fumarate production, was improved by the deletion of the gene that codes for adenylosuccinate synthase (ade12). Similarly, the modification can be studied to improve the acid tolerance of $R$. oryzae. This modification will have a dramatic impact on the feasibility of commercial FA bioproduction strategies as the cost associated with the use of the neutralizing agents as well as the recovery of FA from the fumarate complex formed by the use of a neutralizing agent.

\section{In situ biosynthesis of fumarate esters}

The derivative of the fumaric acid, fumaric acid ester (FAEs), has recently been identified as an important derivative due to its diverse medical applications. Hence, in situ bioconversion of fumaric acid to its ester forms provided an attractive alternative for the green synthesis of the esters. The current methods for industrial synthesis of esters are nonselective, tedious, and consume considerable energy. Most often these commercial processes rely on direct chemical esterification.
This high-temperature inorganic catalyst mediated esterification reactions convert organic acids to esters in the presence of alcohol [88]. Due to the drawbacks of chemical processes, such as non-selectivity and high energy consumption, biosynthesis has been proposed as a suitable alternative.

Lipases are the most used enzymes for the biosynthesis of a diverse group of pharmaceutical compounds. These enzymes have excellent stability even in the presence of organic solvents, hence, their application in the production of diverse biosynthetic compounds. This application of enzymes in the synthesis of a diverse array of compounds has been reviewed extensively by Carvalho et al. [89]. R. oryzae is a known producer of lipases and widely used for industrial applications [90,91]. Hence, it would be possible to bioconvert FA produced to FAEs by inducing lipase synthesis either via genetic modification or induction of their synthesis. The alcohol needed for conversion of FA to FAEs may be provided externally. Ethanol production during $F A$ production fermentation has been reported [40]. The induction of this ethanol 
synthesis would not be recommended as the diversion of sugars to ethanol synthesis can lead to reduced FA production.

\section{Conclusion}

The depletion of the fossil fuels, their impact on the environment and the emphasis on green chemistry has necessitated the production of microorganism mediated synthesis of platform chemicals. This shift allows for the use of the renewable substrate as well as industrial and domestic residues as feedstocks for the manufacture of biochemicals. Due to the low price of commodity chemicals, such as fumaric acid, the main factors that determine economic viability is the costs of substrate and downstream processing. These issues can be overcome by the use of biomass or organic residues as feedstocks for synthesis as well as by employing efficient downstream processing strategies. Hence, the main factor the determines feasibility is the product yield. Maximum product yield is vital to lower manufacturing costs and to make biochemical production competitive against fossil fuelbased production.

Fumaric acid has been identified as one of the key biobased platform chemicals that have diverse applications. Studies to produce this dicarboxylic acid have yielded high titers, especially by the fungus $R$. oryzae, but these high titers obtained are still lower than the theoretical expected yield. Moreover, the complications associated with the use of this fungal strain have necessitated the development of an alternative means for producing this acid. The advances in metabolic engineering tools have allowed for the development of novel microorganisms capable to yield higher fumaric concentration compared to the parental strain. Some of these metabolic engineering strategies that have been investigated and can be adopted for improving FA yield has been discussed in this review. The concentrations achieved are still lower than those obtained by overproducing strains of $R$. oryzae obtained from culture collections. Hence, more research particularly the optimization of the metabolic engineering strategies is essential to obtain higher titers to make bioproduction feasible. This is particularly true of the redirection of metabolic pathways via the introduction of modifications that enable in situ conversion of fumaric acid to its derivatives, such as fumarate esters and aspartic acid. This can potentially improve the feasibility of the bioproduction process.

\section{Disclosure statement}

No potential conflict of interest was reported by the authors.

\section{Funding}

The authors are sincerely thankful for the financial support of the Ministère des Relations Internationales du Québec Q12 (coopération Paraná-Québec 2010-2012) and the Natural Sciences and Engineering Research Council of Canada (NSERC) Discovery Grant.

\section{References}

[1] Bozell JJ, Petersen GR. Technology development for the production of biobased products from biorefinery carbohydrates-the US Department of Energy's "Top 10" revisited. Green Chem. 2010;12.

[2] Choi S, Song CW, Shin JH, et al. Biorefineries for the production of top building block chemicals and their derivatives. Metab Eng. 2015;28:223-239.

[3] Roa Engel CA, Straathof AJJ, Zijlmans TW, et al. Fumaric acid production by fermentation. Appl Microbiol Biotechnol. 2008;78:379-389.

[4] Rodríguez-López J, Sánchez AJ, Gómez DM, et al. Fermentative production of fumaric acid from Eucalyptus globulus wood hydrolyzates. J Chem Technol Biotechnol. 2012;87:1036-1040.

[5] Xu G, Chen X, Liu L, et al. Fumaric acid production in Saccharomyces cerevisiae by simultaneous use of oxidative and reductive routes. Bioresour Technol. 2013; 148:91-96.

[6] Martin-Dominguez V, Estevez J, Ojembarrena F, et al. Fumaric acid production: a biorefinery perspective. Fermentation. 2018;4.

[7] Das RK, Brar SK, Verma M. Fumaric acid. Platform chemical biorefinery. Amsterdam, The Netherlands: Elsevier Inc.; 2016. Available from: http://linkinghub. elsevier.com/retrieve/pii/B9780128029800000080

[8] Das RK, Brar SK, Verma M. Recent advances in the biomedical applications of fumaric acid and its ester derivatives: the multifaceted alternative therapeutics. Pharmacol Rep. 2016;68:404-414.

[9] Doscher CK, Kane JH, Cragwall GO, et al. Industrial applications of fumaric acid. Ind Eng Chem. 1941;33: 315-319.

[10] Reich K, Thaci D, Mrowietz U, et al. Efficacy and safety of fumaric acid esters in the long-term treatment of psoriasis-"A retrospective study (FUTURE). J Dtsch Dermatol Ges. 2009;7:603-610.

[11] Ermis U, Weis J, Schulz JB. PML in a patient treated with fumaric acid. N Engl J Med. 2013;368:1657-1658.

[12] Jansen J, Boerakker MJ, Heuts J, et al. Rapid photocrosslinking of fumaric acid monoethyl ester-functionalized poly(trimethylene carbonate) oligomers for drug delivery applications. J Controlled Release. 2010; 147:54-61.

[13] Jansen J, Tibbe MP, Mihov G, et al. Photo-crosslinked networks prepared from fumaric acid monoethyl ester-functionalized poly(d,I-lactic acid) oligomers and 
[15] Papadaki A, Androutsopoulos N, Patsalou M, et al. Biotechnological production of fumaric acid: the effect of morphology of Rhizopus arrhizus NRRL 2582.

Q5 Fermentation. 2017;3.

[16] GrandViewResearch.com [Internet]. San Francisco [cited 2018 May 10]. Available from: https://www. grandviewresearch.com/industry-analysis/fumaric-acid-

\section{Q6 market.}

[17] IHSMarkit.com [Internet]. London [cited 2018 May 10]. Available from: https://ihsmarkit.com/products/

\section{Q7 fumaric-acid-chemical-economics-handbook.html.}

[18] Goldberg I, Rokem JS, Pines O. Organic acids: old metabolites, new themes. J Chem Technol Biotechnol. 2006;81:1601-1611.

[19] Xu Q, Li S, Huang H, et al. Key technologies for the industrial production of fumaric acid by fermentation. Biotechnol Adv. 2012;30:1685-1696.

[20] Lee JW, Kim HU, Choi S, et al. Microbial production of building block chemicals and polymers. Curr Opin Biotechnol. 2011;22:758-767.

[21] Sauer M, Porro D, Mattanovich D, et al. Microbial production of organic acids: expanding the markets. Trends Biotechnol. 2008;26:100-108.

[22] Roa Engel CA, van Gulik WM, Marang $L$, et al. Development of a low pH fermentation strategy for fumaric acid production by Rhizopus oryzae. Enzyme Microb Technol. 2011;48:39-47.

[23] Zhang K, Zhang L, Yang S-T. Fumaric acid recovery and purification from fermentation broth by activated carbon adsorption followed with desorption by acetone. Ind Eng Chem Res. 2014;53:12802-12808.

[24] Figueira D, Cavalheiro J, Ferreira B. Purification of polymer-grade fumaric acid from fermented spent sulfite liquor. Fermentation. 2017;3.

[25] Yu S, Huang D, Wen J, et al. Metabolic profiling of a Rhizopus oryzae fumaric acid production mutant generated by femtosecond laser irradiation. Bioresour Technol. 2012;114:610-615.

[26] Song CW, Kim DI, Choi S, et al. Metabolic engineering of Escherichia coli for the production of fumaric acid. Biotechnol Bioeng. 2013;110:2025-2034.

[27] Li N, Zhang B, Wang Z, et al. Engineering Escherichia coli for fumaric acid production from glycerol. Bioresour Technol. 2014;174:81-87.

[28] Skory CD, Ibrahim AS. Native and modified lactate dehydrogenase expression in a fumaric acid producing isolate Rhizopus oryzae 99-880. Curr Genet. 2007; 52:23-33.

[29] Wakai S, Arazoe T, Ogino C, et al. Future insights in fungal metabolic engineering. Bioresour Technol. 2017;245:1314-1326.

[30] Abe A, Oda Y, Asano K, et al. Rhizopus delemar is the proper name for Rhizopus oryzae fumaric-malic acid producers. Mycologia. 2007;99:714-722.

[31] Fu Y-Q, Li S, Chen Y, et al. Enhancement of fumaric acid production by Rhizopus oryzae using a two-stage dissolved oxygen control strategy. Appl Biochem Biotechnol. 2010;162:1031-1038.

[32] Huang L, Wei P, Zang R, et al. High-throughput screening of high-yield colonies of Rhizopus oryzae for enhanced production of fumaric acid. Ann Microbiol. 2010;60:287-292.

[33] Kang SW, Lee H, Kim D, et al. Strain development and medium optimization for fumaric acid production. Biotechnol Bioproc E. 2010;15:761-769.

[34] Wen S, Liu L, Nie KL, et al. Enhanced fumaric acid production by fermentation of xylose using a modified strain of Rhizopus arrhizus. BioResources 2013;8.

[35] Liu H, Wang W, Deng L, et al. High production of fumaric acid from xylose by newly selected strain Rhizopus arrhizus RH 7-13-9\#. Bioresour Technol. 2015; 186:348-350.

[36] Liu $\mathrm{H}, \mathrm{Hu} \mathrm{H}$, Jin $\mathrm{Y}$, et al. Co-fermentation of a mixture of glucose and xylose to fumaric acid by Rhizopus arrhizus RH 7-13-9\#. Bioresour Technol. 2017;233: 30-33.

[37] $\mathrm{Xu} \mathrm{Q}, \mathrm{Li} \mathrm{S}, \mathrm{Fu} \mathrm{Y}$, et al. Two-stage utilization of corn straw by Rhizopus oryzae for fumaric acid production. Bioresour Technol. 2010;101:6262-6264.

[38] Deng Y, Li S, Xu Q, et al. Production of fumaric acid by simultaneous saccharification and fermentation of starchy materials with 2-deoxyglucose-resistant mutant strains of Rhizopus oryzae. Bioresour Technol. 2012;107:363-367.

[39] Das RK, Brar SK. Enhanced Fumaric acid production from brewery wastewater and insight into the morphology of Rhizopus oryzae 1526. Appl Biochem Biotechnol. 2014;172:2974-2988.

[40] Das RK, Brar SK, Verma M. A fermentative approach towards optimizing directed biosynthesis of fumaric acid by Rhizopus oryzae 1526 utilizing apple industry waste biomass. Fungal Biol. 2015;119:1279-1290.

[41] Liao W, Liu Y, Frear C, et al. Co-production of fumaric acid and chitin from a nitrogen-rich lignocellulosic material - dairy manure - using a pelletized filamentous fungus Rhizopus oryzae ATCC 20344. Bioresour Technol. 2008;99:5859-5866.

[42] Zhou Y, Nie K, Zhang X, et al. Production of fumaric acid from biodiesel-derived crude glycerol by Rhizopus arrhizus. Bioresour Technol. 2014;163:48-53.

[43] Gu C, Zhou Y, Liu L, et al. Production of fumaric acid by immobilized Rhizopus arrhizus on net. Bioresour Technol. 2013;131:303-307.

[44] Naude A, Nicol W. Fumaric acid fermentation with immobilised Rhizopus oryzae: quantifying time-dependent variations in catabolic flux. Process Biochem. 2017; 56:8-20.

[45] Liu $H$, Zhao $S$, Jin $Y$, et al. Production of fumaric acid by immobilized Rhizopus arrhizus RH 7-13-9\# on loofah fiber in a stirred-tank reactor. Bioresour Technol. 2017;244:929-933.

[46] Das RK, Brar SK, Verma M. Enhanced fumaric acid production from brewery wastewater by immobilization technique. J Chem Technol Biotechnol. 2015;90: 1473-1479.

[47] Cao N, Du J, Gong CS, et al. Simultaneous production and recovery of fumaric acid from immobilized Rhizopus oryzae with a rotary biofilm contactor and 
an adsorption column. Appl Environ Microbiol. 1996; 62:2926-2931.

[48] Gangl IC, Weigand WA, Keller FA. Economic comparison of calcium fumarate and sodium fumarate production byRhizopus arrhizus. Appl Biochem Biotechnol. 1990;24-25:663-677.

[49] Zhou Z, Du G, Hua Z, et al. Optimization of fumaric acid production by Rhizopus delemar based on the morphology formation. Bioresour Technol. 2011;102: 9345-9349.

[50] Ding Y, Li S, Dou C, et al. Production of fumaric acid by Rhizopus oryzae: role of carbon-nitrogen ratio. Appl Biochem Biotechnol. 2011;164:1461-1467.

[51] Meussen BJ, de Graaff LH, Sanders JPM, et al. Metabolic engineering of Rhizopus oryzae for the production of platform chemicals. Appl Microbiol Biotechnol. 2012;94:875-886.

[52] Li D, Tang Y, Lin J, et al. Methods for genetic transformation of filamentous fungi. Microb Cell Fact.

Q10 [53] $\begin{aligned} & 2017 ; 16 . \\ & \text { Dolatabadi S, de Hoog GS, Meis JF, et al. Species }\end{aligned}$ boundaries and nomenclature of Rhizopus arrhizus(syn. $R$. oryzae). Mycoses 2014;57:108-127.

[54] Rhodes RA, Lagoda AA, Misenheimer TJ, et al. Production of fumaric acid in 20-liter fermentors. Appl Microbiol. 1962; 10:9-15.

[55] Kenealy W, Zaady E, Preez JC d, et al. Biochemical aspects of fumaric acid accumulation by Rhizopus arrhizus. Appl Environ Microbiol. 1986; 52:128-133.

[56] $\mathrm{Fu} Y-Q, \mathrm{Xu} Q, \mathrm{Li}$, et al. Strain improvement of Rhizopus oryzae for over-production of fumaric acid by reducing ethanol synthesis pathway. Korean J Chem Eng. 2010;27:183-186.

[57] Zhang B, Skory CD, Yang S-T. Metabolic engineering of Rhizopus oryzae: effects of overexpressing pyc and pepc genes on fumaric acid biosynthesis from glucose. Metab Eng. 2012;14:512-520.

[58] Zhang B, Yang S-T. Metabolic engineering of Rhizopus oryzae: effects of overexpressing fumR gene on cell growth and fumaric acid biosynthesis from glucose. Process Biochem. 2012;47:2159-2165.

Q11 [59] Peleg $Y$, Battat $E$, Scrutton $M$, et al. Isoenzyme pattern and subcellular localisation of enzymes involved in fumaric acid accumulation by Rhizopus oryzae. Appl Microbiol Biotechnol. 1989;32.

[60] Brown SH, Bashkirova L, Berka R, et al. Metabolic engineering of Aspergillus oryzae NRRL 3488 for increased production of I-malic acid. Appl Microbiol Biotechnol. 2013;97:8903-8912.

[61] Lee SY, Lee D-Y, Kim TY. Systems biotechnology for strain improvement. Trends Biotechnol. 2005;23: 349-358.

[62] Toya Y, Shimizu H. Flux analysis and metabolomics for systematic metabolic engineering of microorganisms. Biotechnol Adv. 2013;31:818-826.

[63] Longacre A, Reimers JM, Gannon JE, et al. Flux analysis of glucose metabolism in Rhizopus oryzaefor the purpose of increasing lactate yields. Fungal Genet Biol. 1997;21:30-39.

[64] Bai D-M, Zhao X-M, Li X-G, et al. Strain improvement of Rhizopus oryzae for over-production of $\mathrm{I}(+)$-lactic acid and metabolic flux analysis of mutants. Biochem Eng J. 2004;18:41-48.

[65] Liu Y, Xu Q, Lv C, et al. Study of metabolic profile of Rhizopus oryzae to enhance fumaric acid production under low pH condition. Appl Biochem Biotechnol. 2015;177:1508-1519.

[66] Chen X, Wu J, Song W, et al. Fumaric acid production by Torulopsis glabrata: engineering the urea cycle and the purine nucleotide cycle. Biotechnol Bioeng. 2015;112:156-167.

[67] Raab AM, Lang C. Oxidative versus reductive succinic acid production in the yeast Saccharomyces cerevisiae. Bioeng Bugs 2011;2:120-123.

[68] Zhang T, Wang Z, Deng L, et al. Pull-in urea cycle for the production of fumaric acid in Escherichia coli. Appl Microbiol Biotechnol. 2015;99:5033-5044.

[69] Zhang $T$, Song R, Wang $M$, et al. Regulating C4-dicarboxylate transporters for improving fumaric acid production. RSC Adv. 2017;7:2897-2904.

[70] Xu G, Liu L, Chen J. Reconstruction of cytosolic fumaric acid biosynthetic pathways in Saccharomyces cerevisiae. Microb. Cell Fact. 2012;11.

[71] $\mathrm{Xu} \mathrm{G}, \mathrm{Wu} M$, Jiang L. Site-saturation engineering of proline 474 in pyruvate carboxylase from Rhizopus oryzae to elevate fumaric acid production in engineered Saccharomyces cerevisiae cells. Biochem Eng J. 2017;117:36-42.

[72] Chen $X$, Song $W$, et al. Fumarate production by Torulopsis glabrata: engineering heterologous fumarase expression and improving acid tolerance. PLoS One. 2016;11.

[73] Wang G, Huang D, Qi H, et al. Rational medium optimization based on comparative metabolic profiling analysis to improve fumaric acid production. Bioresour Technol. 2013;137:1-8.

[74] Gu S, Xu Q, Huang H, et al. Alternative respiration and fumaric acid production of Rhizopus oryzae. Appl Microbiol Biotechnol. 2014;98:5145-5152.

[75] Michielse CB, Salim K, Ragas P, et al. Development of a system for integrative and stable transformation of the zygomycete Rhizopus oryzae by Agrobacteriummediated DNA transfer. Mol Genet Genomics. 2004; 271:499-510.

[76] Zubieta MP, da Silva Coelho I, de Queiroz MV, et al. Agrobacterium tumefaciens-mediated genetic transformation of the ectomycorrhizal fungus Laccaria laccata. Ann Microbiol. 2014;64:1875-1878.

[77] Zhong $\mathrm{YH}$, Wang $\mathrm{XL}$, Wang $\mathrm{TH}$, et al. Agrobacteriummediated transformation (AMT) of Trichoderma reesei as an efficient tool for random insertional mutagenesis. Appl Microbiol Biotechnol. 2007;73:1348-1354.

[78] Nyilasi I, Papp T, Csernetics Á, et al. Agrobacterium tumefaciens-mediated transformation of the zygomycete fungus Backusella lamprospora. J Basic Microbiol. 2008;48:59-64.

[79] Lee LY, Gelvin SB. T-DNA binary vectors and systems. Plant Physiol. 2007;146:325-332.

[80] Deng H, Gao R, Liao X, et al. CRISPR system in filamentous fungi: current achievements and future directions. Gene. 2017;627:212-221. 


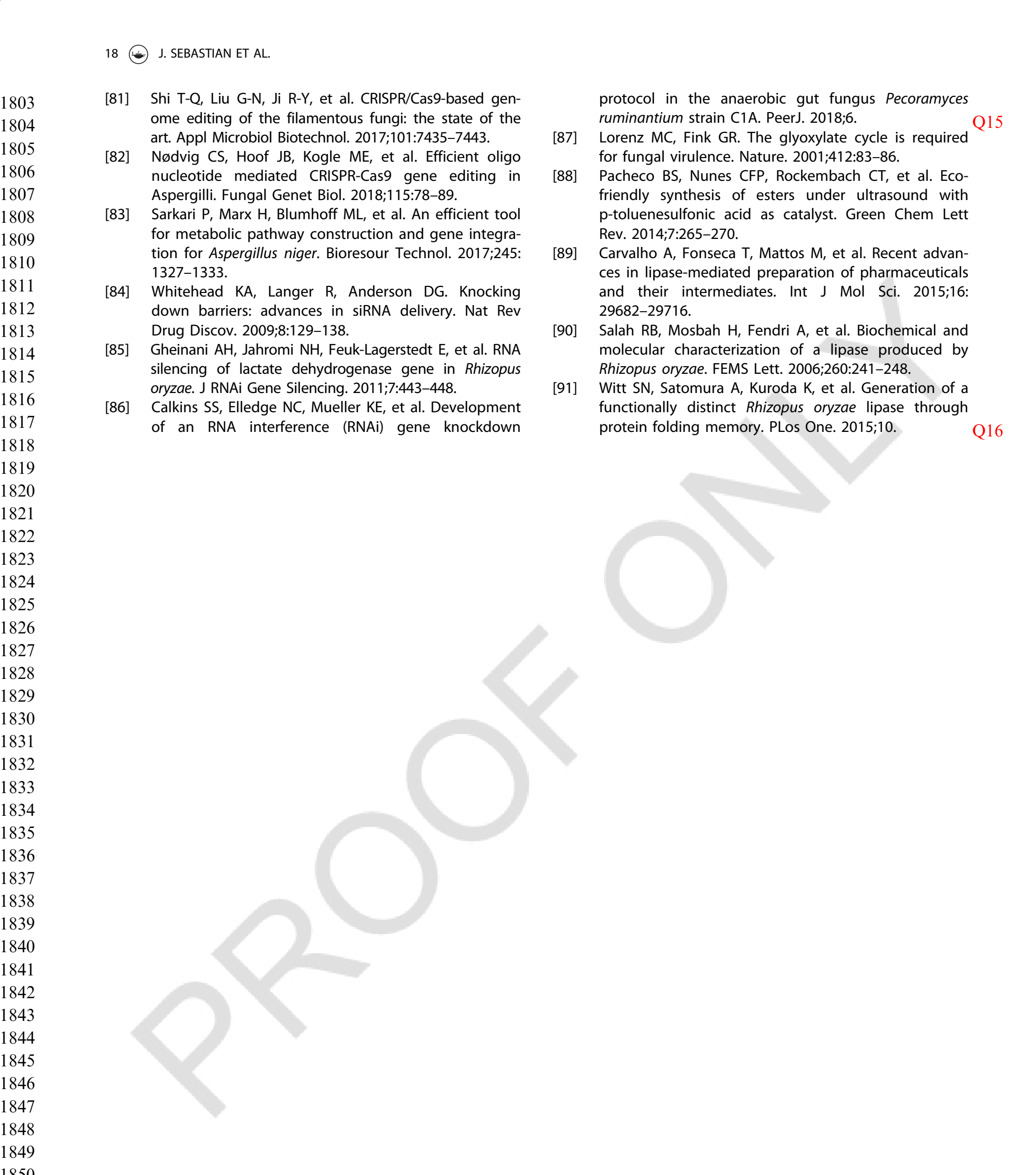

